\title{
Effect of bars on the galaxy properties
}

\author{
Matias Vera ${ }^{1}$, Sol Alonso ${ }^{2}$, and Georgina Coldwell ${ }^{2}$ \\ 1 ICATE, UNSJ-CONICET, CC49, 5400 San Juan, Argentina \\ e-mail: solalonsog@gmail.com \\ 2 Facultad de Ciencias Exactas, Físicas y Naturales, UNSJ-CONICET, 5400 San Juan, Argentina \\ Received 20 April 2016 / Accepted 27 July 2016
}

\begin{abstract}
Aims. With the aim of assessing the effects of bars on disk galaxy properties, we present an analysis of different characteristics of spiral galaxies with strong bars, weak bars and without bars.

Methods. We identified barred galaxies from the Sloan Digital Sky Survey (SDSS). By visual inspection of SDSS images we classified the face-on spiral galaxies brighter than $g<16.5$ mag into strong-bar, weak-bar, and unbarred galaxies. With the goal of providing an appropriate quantification of the influence of bars on galaxy properties, we also constructed a suitable control sample of unbarred galaxies with similar redshifts, magnitudes, morphology, bulge sizes, and local density environment distributions to those of barred galaxies.

Results. We found 522 strong-barred and 770 weak-barred galaxies; this represents a bar fraction of $25.82 \%$ with respect to the full sample of spiral galaxies, in good agreement with several previous studies. We also found that strong-barred galaxies show lower efficiency in star formation activity and older stellar populations (as derived with the $D_{n}(4000)$ spectral index) with respect to weakbarred and unbarred spirals from the control sample. In addition, there is a significant excess of strong-barred galaxies with red colors. The color-color and color-magnitude diagrams show that unbarred and weak-barred galaxies are more extended towards the blue zone, while strong-barred disk objects are mostly grouped in the red region. Strong-barred galaxies present an important excess of high metallicity values compared to unbarred and weak-barred disk objects, which show similar $12+\log (\mathrm{O} / \mathrm{H})$ distributions. Regarding the mass-metallicity relation, we found that weak-barred and unbarred galaxies are fitted by similar curves, while strongbarred ones show a curve that falls abruptly with more significance in the range of low stellar masses $\left(\log \left(M_{*} / M_{\odot}\right)<10.0\right)$. These results would indicate that prominent bars produced an accelerating effect on the gas processing, reflected in the significant changes in the physical properties of the host galaxies.
\end{abstract}

Key words. galaxies: spiral - galaxies: formation - galaxies: evolution

\section{Introduction}

Galactic bars are structures observed in a significant fraction of spiral galaxies and are believed to have an important role in the dynamical evolution of their hosts. Several simulations show that bars can efficiently transport gas from the outer zones to the innermost central regions of the barred galaxies (Weinberg 1985; Debattista \& Sellwood 1998; Athanassoula 2003). By interaction with the edges of the bars, the gas clouds suffer shocks producing angular momentum losses and allowing a flow of material toward central kiloparcec scale (Shlosman et al. 1990). Moreover, some works show that bars can be destroyed by a large central mass concentration (Roberts et al. 1979; Norman et al. 1996; Sellwood \& Moore 1999; Athanassoula et al. 2005). This finding indicates that disk galaxies that are currently nonbarred possibly had a bar in the past (Kormendy \& Kennicutt 2004) and also that bars may be recurrent in the galaxy life (Bournaud \& Combes 2002; Berentzen et al. 2004; Gadotti \& Souza 2006), which means that in this context bars formed at different times, and with different conditions, might be present in the barred disk galaxies.

Owing to the high efficiency of gas inflow, galactic bars can alter several properties of disk galaxies on relatively short timescales. In this sense, the presence of bars can affect the star formation activity, stellar population, and colors, can modify the galactic structure (Athanassoula 1983; Buta \& Combes 1996), and can change the chemical composition
(Combes et al. 1993; Martin 1995), contributing to the evolution process of the host galaxies (Ellison et al. 2011; Zhou et al. 2015). In addition, the inflow processes are also considered an efficient mechanism for triggering active galactic nuclei (AGN) (Combes et al. 1993; Corsini et al. 2003; Alonso et al. 2013, 2014), and forming bulges or pseudo-bulges (e.g., Combes \& Sanders 1981; Kormendy \& Kennicutt 2004; Debattista et al. 2005, 2006; Martinez-Valpuesta et al. 2006; Méndez-Abreu et al. 2008; Aguerri et al. 2009).

With respect to the relation between bars and host galaxy colors from statistical analysis, different studies show diverse results. Several observational works have found that the bar fraction, $f_{\text {bar }}$, is higher in later-type spiral galaxies that are bluer and less concentrated systems (e.g., Barazza et al. 2008; Aguerri et al. 2009). However, other studies have found an excess of barred galaxies with redder colors from different samples. Masters et al. (2010aa) have found a high fraction of bars in passive red spiral galaxies for a sample obtained from the Galaxy Zoo catalog (Lintott et al. 2011). In addition, Oh et al. (2012) have shown that a significant number of barred galaxies are redder than their counterparts of unbarred spiral galaxies. In our previous works (Alonso et al. 2013, 2014) we found an excess of red colors in spiral barred AGN with respect to unbarred active galaxies in a suitable control sample.

The role of the bars in star formation and metallicity is the subject of several works; however the conclusions are not clear. Many studies found that bars enhanced the star formation 
rates (SFR) in spiral galaxies compared to unbarred ones (e.g., Hawarden et al. 1986; 1996; Devereux 1987; Hummel 1990), while other works show that bars do not guarantee an increase in star formation activity (Pompea \& Rieke 1990; Martinet \& Friedli 1997; Chapelon et al. 1999). In a similar way, different authors found diverse results in the metalicity studies in barred galaxies with respect to their unbarred counterparts (e.g., Vila-Costas \& Edmunds 1992; Oey \& Kennicutt 1993; Martin \& Roy 1994; Zaritsky et al. 1994; Considere et al. 2000; Ellison et al. 2011). More recently, using data from the CALIFA survey, Sánchez-Blázquez et al. (2014) have performed a comparative study of the stellar metallicity and age gradients in a sample of 62 spiral galaxies finding no differences between the presence or absence of bars.

The discrepancy in the results of the bar effects on SFR and metallicity may depend on the host galaxy morphology (Huang et al. 1996; Ho et al. 1997; James et al. 2009) and may be also due to the length/strength of the bar (Elmegreen \& Elmegreen 1985, 1989; Erwin 2004; Menendez-Delmestre et al. 2007). Similarly, some studies (e.g., Athanassoula 1992; Friedli et al. 1994; Friedli \& Benz 1995) from numerical simulations found such trends, showing that bar strength is related to the efficiency and quantity of gas inflow, and therefore to the star formation activity and metallicity gradients.

Furthermore, different authors have proposed diverse ways to build control samples from unbarred galaxies, and have used them to obtain conclusions from comparative studies, and so the discrepancy in the results could be due to a biased selection of these samples. In this direction, Perez et al. (2009a,b) have found that a control sample of interacting galaxies should be selected matching, at least, redshift, morphology, stellar masses, and local density environment. These are also a suitable criteria for building control samples of barred galaxies (Alonso et al. 2013, 2014). Motivated by these finds, in this paper we conducted a detailed analysis of the effect of bars on host galaxy properties, with respect to the unbarred ones by studying different characteristics (e.g., color, stellar population, star formation activity, metallicity) with the aim of assessing whether bar structure in disks plays a significant role in modifying galaxy properties, and how strong this effect is.

This paper is structured as follows. Section 2 presents the procedure used to construct the catalog of barred galaxies from the Sloan Digital Sky Survey (SDSS), the classification of the bar structures, and the control sample selection criteria. In Sect. 3, we explore different properties of the barred spirals in comparison with unbarred galaxies obtained from a suitable control sample. We analyze in detail the influence of bars on star formation activity, stellar population, color indexes, and metallicity in host spiral galaxies with respect to unbarred ones. Finally, Sect. 4 summarizes our main results and conclusions. The adopted cosmology through this paper is $\Omega=0.3, \Omega_{\lambda}=0.7$, and $H_{0}=$ $100 \mathrm{~km} \mathrm{~s}^{-1} \mathrm{Mpc}$.

\section{Catalog of barred galaxies}

The analysis of this paper is based on the Sloan Digital Sky Server Data Release 7 (SDSS-DR7, Abazajian et al. 2009). It uses a $2.5 \mathrm{~m}$ telescope to obtain photometric and spectroscopic data which cover nearly one-quarter of the celestial sphere and collect spectra of more than one million objects. DR7 includes 11663 square degrees of sky imaged in five wavebands $(u, g, r, i, z)$ containing the photometric parameters of 357 million objects. The main galaxy sample, which contains about 900000 galaxies with measured spectra and photometry, is essentially a magnitude-limited spectroscopic sample $r_{\lim }<$ 17.77 (Petrosian magnitude), and most of galaxies span a redshift range $0<z<0.25$ with a mean readshift of 0.1 (Strauss et al. 2002). For this work, several physical properties of galaxies were derived and published for the SDSS-DR7 galaxies: gas-phase metallicities, stellar masses, current total and specific star formation rates, concentration index, etc. (Brinchmann et al. 2004; Tremonti et al. 2004; Blanton et al. 2005). These data were obtained from the MPA/JHU ${ }^{1}$ and the NYU ${ }^{2}$ added-values catalogs.

With the aim of obtaining barred galaxies, we first crosscorrelated the SDSS galaxies with the spiral objects obtained from the Galaxy Zoo catalog (Lintott et al. 2011), which comprises a morphological classification of nearly 900000 galaxies drawn from the SDSS. In order to cover a wide coverage area, hundreds of thousands of volunteers contribute to this survey; however, the large number of classifiers makes it difficult to maintain a unified criteria. They define different categories (elliptical, spiral, merger, uncertain, etc.) and give the fraction of votes in each category. In this study, we selected galaxies that were classified as spiral objects by the Galaxy Zoo team with a fraction of votes $>0.6$. Taking this into account, a low fraction of galaxies with non-spiral morphological types could be included. In addition, we exclude AGN objects (Coldwell et al. 2014), whose emission line spectral features could affect our interpretation of the results. Furthermore, as bars are objects that lie on the host disk plane (Sellwood \& Wilkinson 1993) and visual inspection become less efficient while inclination increases, we applied another restriction on the ellipticity of the objects, selecting galaxies with axial ratio $b / a>0.4$. We also restricted the spiral edge-on galaxy sample in redshift $(z<0.06)$ and imposed a magnitude cut so that the extinction corrected SDSS $g$-mag is brighter than 16.5. With these restrictions, our sample comprises 6771 galaxies, and, therefore, we can make a plausible visual inspection of a good set of objects.

\subsection{Classification}

We proceeded to select barred galaxies by visual inspection. For this task, we used the $g+r+i$ combined color images obtained from the online SDSS-DR7 Image Tool ${ }^{3}$. Then, by a detailed visual examination we classified the galaxies into four groups based on the presence of bars, taking into account their relative light contribution and length with respect to the structural properties of the host galaxies. We can summarize the classification as follows: 522 strong-barred galaxies (where the size of the bars is at least $30 \%$ of their host galaxy sizes), 770 weakbarred galaxies (the size of the bars is less than $30 \%$ the size of their host galaxies), 688 ambiguous-barred galaxies (objects for which it is difficult to decide whether there is a bar or not), and 3711 non-barred galaxies. We also found some galaxies with elliptical and irregular appearance, which were removed from our sample. The details of the classification are listed in Table 1, and Fig. 1 shows examples of each galaxy type studied in this work.

Therefore, the final catalog consists of 1292 spiral objects with strong and weak bars (we excluded ambiguous-barred galaxies that did not completely match the bar classification). This represents a fraction of $25.82 \%$ with respect to the sample of 5003 spiral galaxies with clear classification. In the

\footnotetext{
http://www . mpa-garching.mpg.de/SDSS/DR7/

http://sdss.physics.nyu.edu/vagc/

http://skyserver.sdss.org/dr7/en/tools/chart/list. asp
} 
Table 1. Galaxy classification, numbers and percentages of objects.

\begin{tabular}{ccc}
\hline \hline Galaxy type & $N^{0}$ of objects & Percentages \\
\hline Non-barred galaxies & 3711 & $54.80 \%$ \\
Strong-barred galaxies & 522 & $7.71 \%$ \\
Weak-barred galaxies & 770 & $11.37 \%$ \\
Ambiguous-barred galaxies & 688 & $10.16 \%$ \\
Elliptical and irregular galaxies & 1080 & $15.96 \%$ \\
Total sample & 6771 & $100.0 \%$ \\
\hline
\end{tabular}

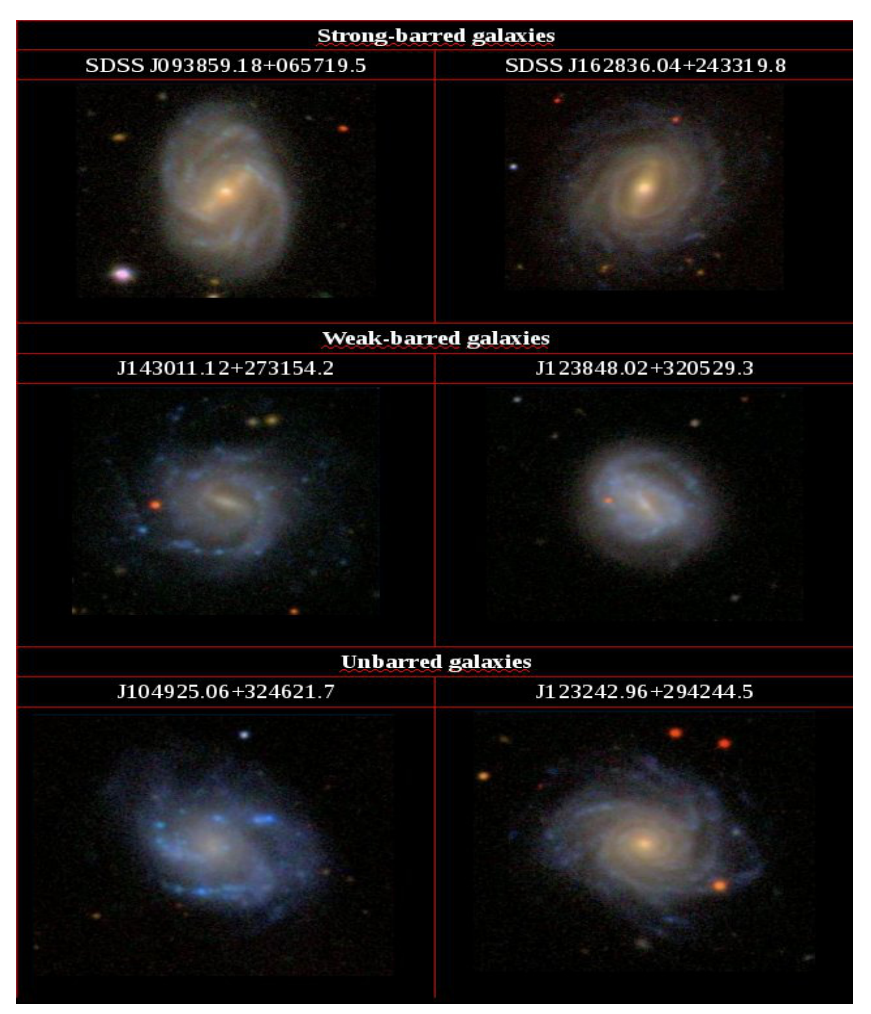

Fig. 1. Images of typical examples of galaxies classified as strong and weak-barred objects (upper and medium panels, respectively). Lower panels show examples of spirals without bar.

same direction, several works carried out by means of visual inspection of different galaxy samples (e.g., the RC3 and UGC; de Vaucouleurs et al. 1991; Nilson 1973; Marinova et al. 2009; Alonso et al. 2013) find a bar fraction of $25-30 \%$ in agreement with this work.

\subsection{Control sample}

To provide a suitable quantification of the impact of bars on the host galaxy properties, we obtained a reliable control sample of unbarred disk objects following Alonso et al. (2013).

We constructed a control sample of galaxies using a Monte Carlo algorithm that selected objects classified as unbarred galaxies in the previous section with similar redshift and extinction and $K$-corrected (Blanton et al. 2003) absolute $r$-band Petrosian magnitude distributions of the barred galaxy sample (see panels a and b in Fig. 2).

We also considered unbarred galaxies in the control sample with similar concentration index $(C)^{4}$ distribution to that of the barred catalog to obtain a similar bulge-to-disk ratio in both samples (panel c in Fig. 2). Furthermore, we restricted the control

\footnotetext{
$C=r 90 / r 50$ is the ratio of Petrosian $90 \%-50 \% r$-band light radii.
}

unbarred spirals to match the fracdeV parameter defined as the fraction of the light fit by a de Vaucouleurs profile over an exponential profile, where a pure de Vaucouleurs elliptical should have fracdeV $=1$ and a pure exponential disk spiral will have fracdeV $=0$ (Masters et al. 2010aa). Thus, this index estimates the surface brightness distribution of the bulge, so that it is a good indicator of its size in galaxies with disk morphology (Kuehn 2005; Bernardi et al. 2010; Skibba et al. 2012, see panel $d$ in Fig. 2). Therefore, the possible differences in the results are associated with the presence of bars and not with the difference in the bulge prominences or with the global galaxy morphology.

In addition, in order to obtain galaxies in the same density regions, we also selected objects without bars with a similar distribution of the local density environment parameter $\left(\Sigma_{5}\right)$ to that of barred galaxies. This parameter is calculated through the projected distance $d$ to the fifth nearest neighbor galaxy, $\Sigma_{5}=5 /\left(\pi d^{2}\right)$, brighter than $M_{r}=-20.5$, and within a radial velocity difference of less than $1000 \mathrm{~km} \mathrm{~s}^{-1}$ (Balogh et al. 2004). Figure 2 (panel e) shows the distributions of the $\log \left(\Sigma_{5}\right)$ for both samples.

With these restrictions we obtained a control sample of 2205 unbarred spiral objects with similar redshift, brightness, morphology, bulge prominence, and local environment to that of barred galaxies. Then, any difference in the galaxy properties is associated only with the presence of the bar and, consequently, by comparing the results we estimate the real difference between barred and unbarred galaxies, unveiling the effect of this structure on the disk galaxy features.

\section{Galaxy properties}

Different studies have shown that bars can induce several processes that modify many properties of the galaxies (Sellwood \& Wilkinson 1993; Combes et al. 1993; Zaritzky et al. 1994; Lee et al. 2012; Oh et al. 2012; Alonso et al. 2013, 2014). However, there are still many questions about how the properties are modified by the presence of a bar structure in the disk of the spiral galaxies.

In this section we explore the effect of bars, with different structural strength, on the stellar population, star formation activity, colors, and metallicity of the host galaxies, in comparison with the unbarred objects in a suitable control sample obtained from the previous section. This analysis may help to deepen our understanding of this issue which has been explored by different authors using diverse approaches.

\subsection{Star formation and stellar population}

With the aim of assessing the effect of bars on the star formation and stellar age population, in the following analysis we use the specific star formation rate parameter, $\log \left(S F R / M_{*}\right)$, as a good indicator of the star formation activity in non-AGN galaxies. It is estimated as a function of the $\mathrm{H} \alpha$ line luminosity, and normalized using stellar masses (Brinchmann et al. 2004). We also employ the spectral index $D_{n}(4000)$ (Kauffmann et al. 2003), which estimates the age of stellar populations. It is calculated from the spectral discontinuity occurring at $4000 \AA$ produced by an accumulation of a large number of spectral lines in a narrow region of the spectrum, especially important in old stars. In this analysis, we use the $D_{n}(4000)$ definition obtained by Balogh et al. (1999), as the ratio of the average flux densities in the nar-

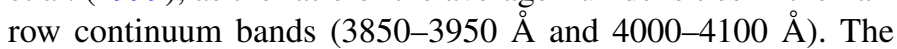



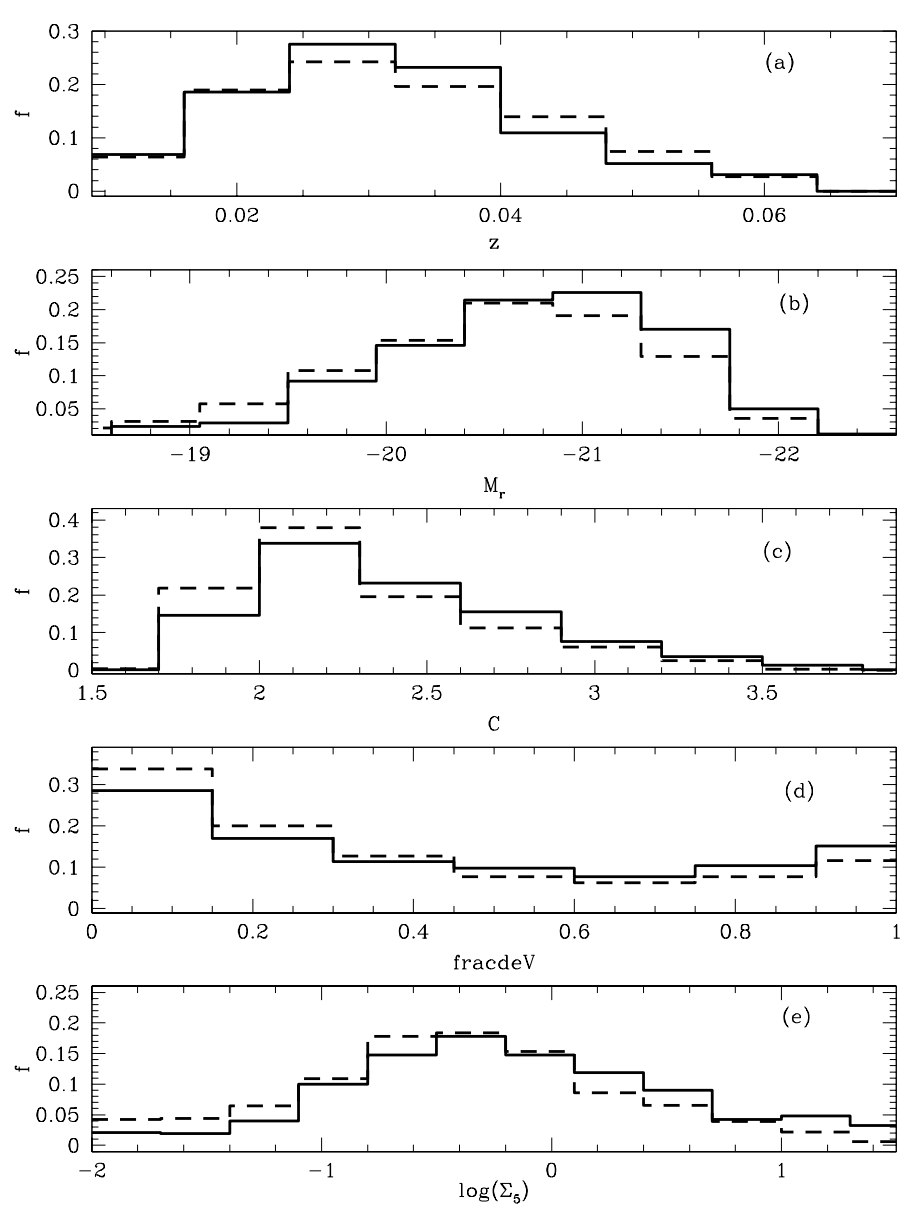

Fig. 2. Distributions of redshift, luminosities, concentration index, bulge size indicator, and local density parameter, $z, M_{r}, C$, fracdeV, and $\log \left(\Sigma_{5}\right)$ (panels a), b, c), d), and e)), for barred galaxies (solid lines) and galaxies without bars in the control sample (dashed lines).

spectroscopic data in SDSS are obtained within the aperture of a spectroscopic fiber ( 3 arcsec in diameter). This corresponds to a typical physical size of the fiber of $\approx 2 \mathrm{kpc}$ at the mean redshift of our sample $(z \approx 0.03)$. Nevertheless, the SDSS spectroscopic parameters (e.g., SFR and metallicity) outside of the fiber are estimated following different methodologies, using the galaxy photometry (see Kauffmann et al. 2003; Tremonti et al. 2004; and Brinchmann et al. 2004, for details).

In Fig. 3 (upper panel), we show the star formation activity distributions for each classified galaxy type. It can be clearly seen that strong-barred galaxies show a significant excess toward lower $\log \left(S F R / M_{*}\right)$ values with respect to weak-barred and unbarred objects in the control sample. In addition, a remarkable bimodality can be observed in galaxies with strong bar prominences. This behavior clearly shows that there is an excess of strong-barred galaxies with low star formation activity, indicating that the amount of gas may be not sufficient, after being consumed by star formation in the previous process during the prior stages of the galaxy life. The value located near $\log \left(S F R / M_{*}\right) \approx-11.3$ divides both distributions. Furthermore, weak-barred and unbarred galaxies show a similar distribution of specific star formation rate.

Moreover, while several barred galaxies have more concentrated $\mathrm{CO}$ in the central region, some early-type disks have a lack of CO in this region (e.g., Sheth et al. 2005; Sakamoto et al. 1999). In this sense, Sheth et al. (2005), using CO observations of the six barred spirals, finding that their sample of barred
Table 2. Percentages of spiral galaxies with strong bars, weak bars, and without bars with $\log \left(S F R / M_{*}\right)>-11.3$ and $D_{n}(4000)<1.8$.

\begin{tabular}{ccc}
\hline \hline Type & $\log \left(S F R / M_{*}\right)>-11.3$ & $D_{n}(4000)<1.8$ \\
\hline Strong-barred & $79 \%$ & $77 \%$ \\
Weak-barred & $97 \%$ & $97 \%$ \\
Unbarred & $93 \%$ & $91 \%$ \\
\hline
\end{tabular}

galaxies have very little molecular gas in the central regions of the galaxies. It could indicate that the gas was consumed in star formation processes at an earlier stage of the galaxy evolution. More recently, James \& Percival (2016) studied the central regions of four barred galaxies, showing that the star formation activity is inhibited within each of these galaxies, suggesting that star formation appears to have been suppressed by the bar.

The lower panel in Fig. 3 shows the distributions of the spectral index $D_{n}(4000)$ for spiral galaxies in the different samples. As we can see, strong-barred galaxies show an important excess toward higher $D_{n}(4000)$ values in comparison with weak-barred and unbarred spiral objects, indicating that strong bars tend to exist in host galaxies with older stellar population. This finding could suggest that strong bars preferentially formed in galaxies with old stellar population or that strong bars formed a long time ago and thus the stellar populations of galaxies became old. In this direction, Sheth et al. (2008) show that bars were formed first in massive and luminous galaxies, and later less massive and bluer systems acquired the majority of their bars. Therefore, the strong-bar excess toward higher $D_{n}(4000)$ values could represent bars formed in the first stage that have grown together with their hosts. In addition, different authors (e.g., Weinzirl et al. 2009; Laurikainen et al. 2007, 2009) show that, in general, strong bars are more frequently found in early-type disk galaxies which are more massive than late-type objects. Similar to what is observed in the star formation distributions, strong-barred galaxies show a bimodality in the stellar population around $D_{n}(4000) \approx 1.8$. Table 2 quantifies the percentages of galaxies in our different samples with efficient star formation activity and a young stellar population. In a similar way, Sanchez-Blazquez et al. (2011) found an old stellar population in four barred galaxies. This is in agreement with the findings of James \& Percival (2016) from the spectroscopic analysis in four different disk objects. In addition, old stellar populations have also been found in galaxies with bars by several studies (Perez et al. 2007, 2009a; de Lorenzo-Caceres et al. 2012, 2013), in agreement with our result.

In addition, we divide strong-barred galaxies into two subsamples: a group of galaxies that belong to the minor peak of the distributions of both $\log \left(S F R / M_{*}\right)$ and $D_{n}(4000)$ (Group 1, G1) and a group of strong-barred galaxies belonging to the major peak distributions in Fig. 3 (Group 2, G2). The limits distinguishing the two groups are $\log \left(S F R / M_{*}\right)=-11.3$ and $D_{n}(4000)=1.8$. In this way, we can reveal whether the differences in galaxy properties are mainly driven by the different star formation and stellar population or because they have a bar.

We checked disk galaxies in the smaller peak in the previous bimodal distributions with older stellar population and low efficiency in star formation activity. We noticed that there are 97 strong-barred lenticular galaxies. In Fig. 4 some examples can be seen. Therefore, an important fraction (about the 20\%) of strong-barred galaxies are SB0 morphological types, which could indicate that when a lenticular galaxy contains a bar, it is ususally an strong structure. These results are consistent with those obtained by Aguerri et al. (2009), who found that bar 
M. Vera et al.: Effect of bars on the galaxy properties
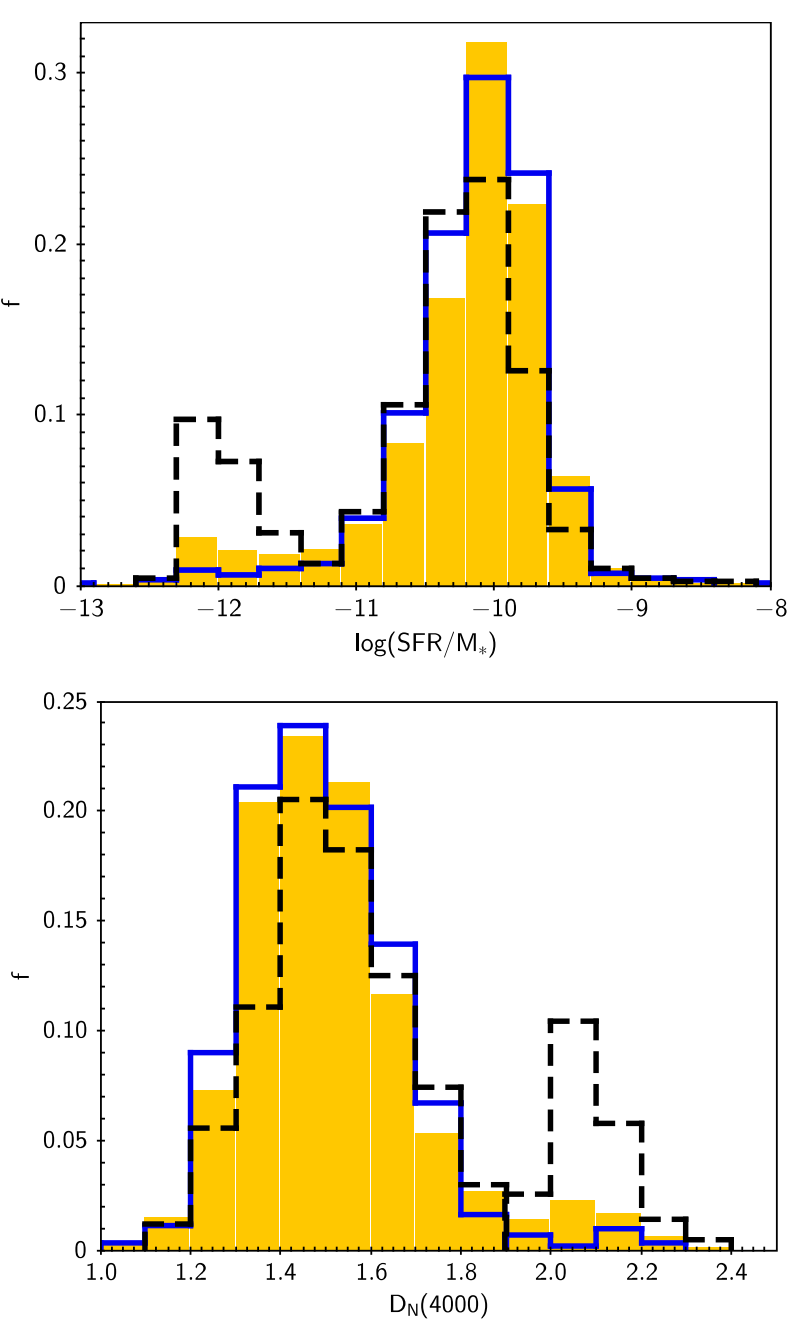

Fig. 3. Specific star formation rate, $\log \left(S F R / M_{*}\right)$ (upper panel) and stellar population, $D_{n}(4000)$ (lower panel) distributions for different galaxy types: unbarred objects (shaded histograms), weak-barred (solid lines) and strong-barred (dashed lines) galaxies.
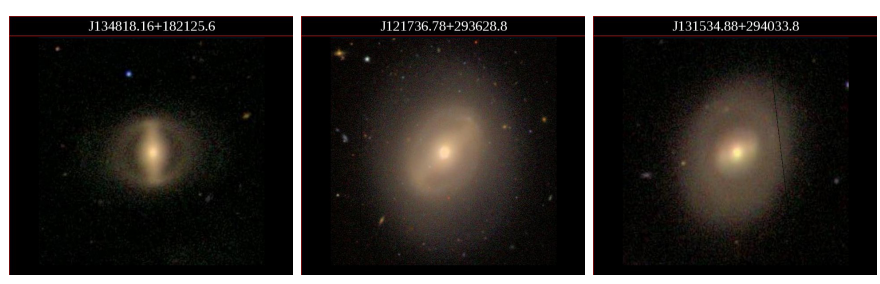

Fig. 4. Three examples of barred lenticular galaxies with $\log \left(S F R / M_{*}\right)<-11.3$ and $D_{n}(4000)>1.8$.

length (normalized by the galaxy size) in lenticular galaxies tend to be longer than in late-types objects. Furthermore, our finding agrees with Laurikainen et al. (2009), who found that prominent bars (calculated using the maximum $m=2$ Fourier density amplitude) are more common in lenticular galaxies than weak bars (considering that lenticular galaxies in their work are subdivided into S0 and S0/a types, and that a medium amplitude is defined between strong and weak structures).

In order to understand the behavior of star formation and stellar populations of spirals with strong and weak bars and without bars with respect to the stellar masses and the morphology of the host galaxy, we have analyzed $\log \left(S F R / M_{*}\right)$ and $D_{n}(4000)$ as a function of $\log \left(M_{*}\right)$ and concentration index, $C$. Figure 5
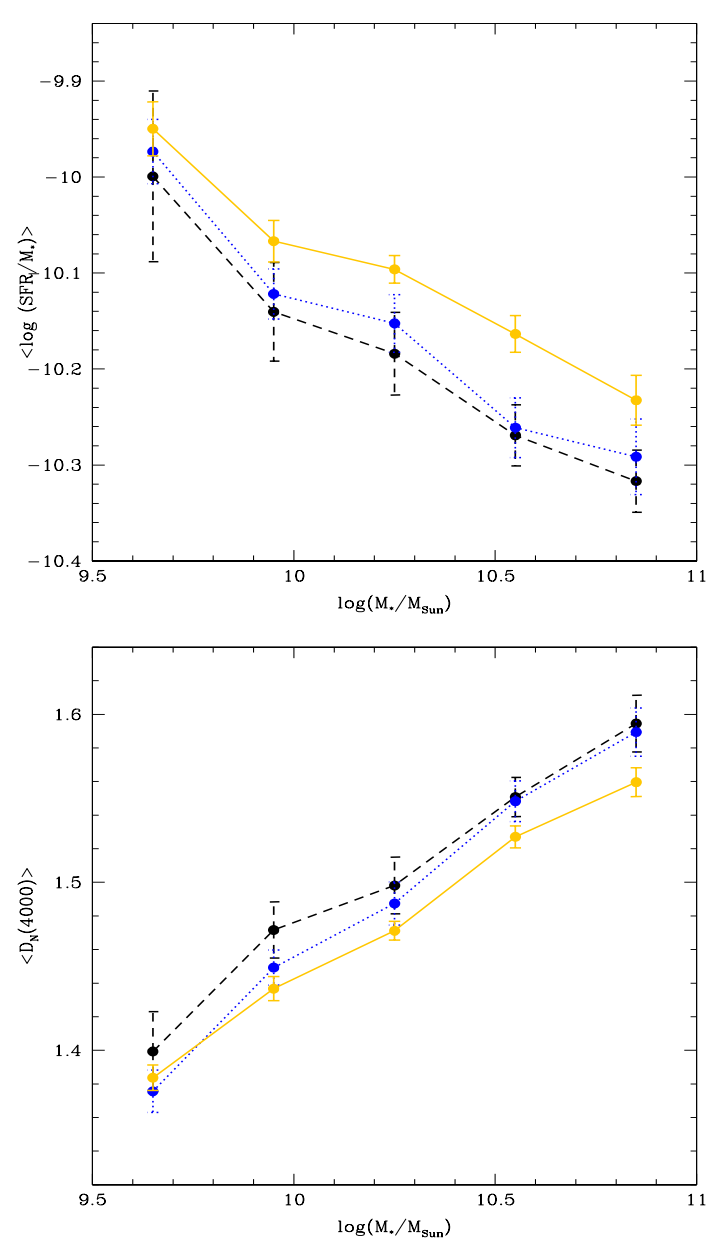

Fig. 5. Specific star formation rate, $\log \left(S F R / M_{*}\right)$, and $D_{n}(4000)$ (upper and lower panels) as a function of stellar mass bins for unbarred, weak and strong-barred spiral galaxies that belong to the major peaks of the star formation and stellar population distributions (see Fig. 3) (solid, dotted, and dashed lines, respectively).

shows the mean $\log \left(S F R / M_{*}\right)$ and $D_{n}(4000)$ as a function of the stellar mass. Errors were estimated by applying the bootstrap resampling technique in all figures (Barrow et al. 1984). For the Figs. 5 and 6 we considered barred galaxies that belong to the major peaks in Fig. 3, with the aim of excluding the fraction of the lenticular galaxies. As can be seen, star formation activity decreases towards higher stellar masses and, in the same direction, young stellar population diminish with $\log \left(M_{*}\right)$. Clearly host galaxies with strong bars show a systematically lower efficiency of star formation activity and an older stellar population in all stellar mass bins with respect to the other samples studied in this paper. Furthermore, disk objects in the control sample show efficient activity in star formation and younger stellar population. In addition, the trends for weak-barred galaxies are observed between strong-barred objects and the control sample.

In addition, in Fig. 6 we can clearly see that strongbarred galaxies become less efficient star formers whith increasing the $C$ index. On the other hand, for unbarred and weakbarred galaxies $\log \left(S F R / M_{*}\right)$ remains almost constant for the all $C$ values. Moreover, strong-barred objects show older stellar populations towards galaxies with earlier morphology. Unbarred spirals show younger stellar age populations and higher SFR values for different morphological types, while weak-barred galaxies present intermediate tendencies. This could indicate that bars tend to modify their host galaxy properties quickly (i.e., bars 

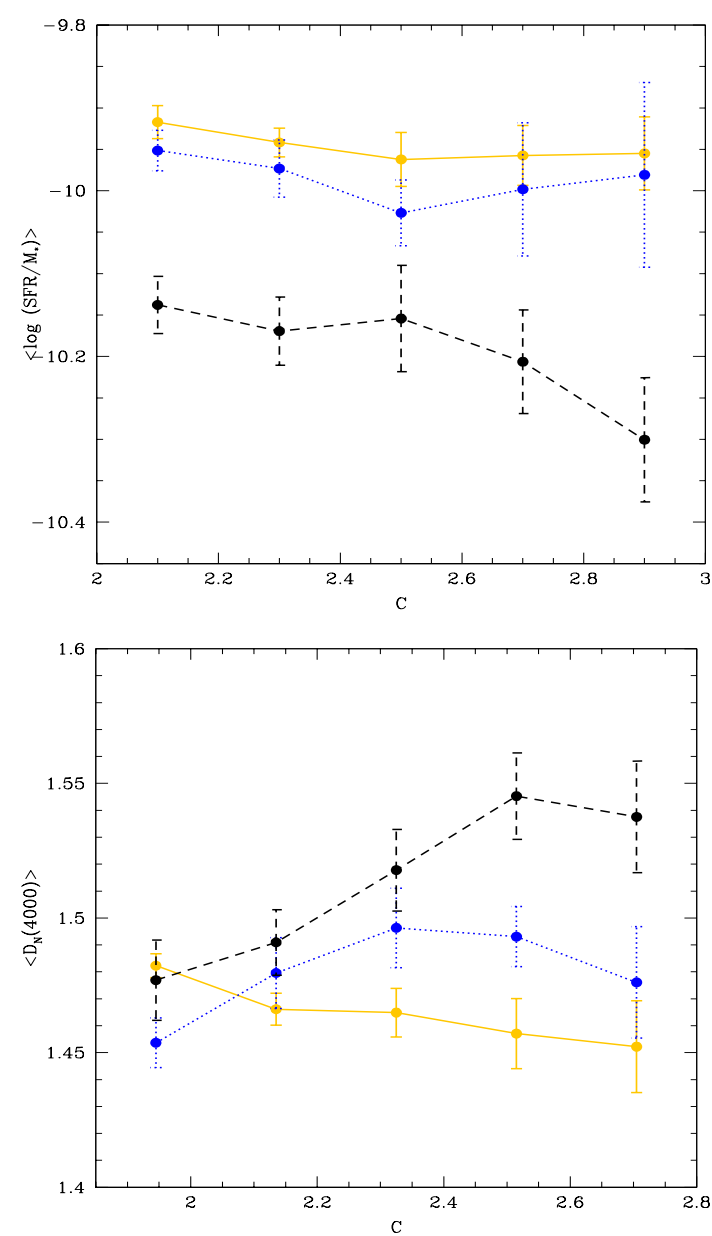

Fig. 6. Specific star formation rate $\left(\log \left(S F R / M_{*}\right)\right.$, upper panel $)$ and stellar age population $\left(D_{n}(4000)\right.$, lower panel $)$ as a function of parameter $C$ for unbarred, weak-barred, and strong-barred galaxies that belong to the major peaks of the star formation and stellar population distributions (see Fig. 3) (solid, dotted, and dashed lines, respectively).

could accelerate the gas processing) when they have became prominent enough.

In the same direction, Masters et al. (2012) found a significantly lower bar fraction on the gas-rich galaxies with respect to gas-poor disk objects. The authors suggest that in gasrich disks the bars funnel the gas into the central region of the galaxy. Then, this material can turn into molecular gas and eventually trigger star formation acticity (e.g., Ho et al. 1997; Sheth et al. 2005; Ellison et al. 2011; Lee et al. 2012). This process could then accelerate the gas consumption, ceasing the formation of the new stars by removing gas from the outer regions of the disk, and become red host galaxies. These mechanisms may indicate different evolutionary stages of the bars in spiral galaxies, which depend on the strength of the bar structure. In this context, Jogee et al. (2005), based on the properties of circumnuclear gas and star formation, proposed a possible scenario of bar-driven dynamical evolution of the galaxies. In the first phases, large amounts of gas are transported by the bars towards the galactic central regions, along with an efficient star formation activity. Then, in the post-starburst phase, the gas is consumed by circumnuclear starburst, showing low SFR (Sheth et al. 2005). In this context, we argue that during these different stages, the length/strength of the bars and host galaxy properties are modified.
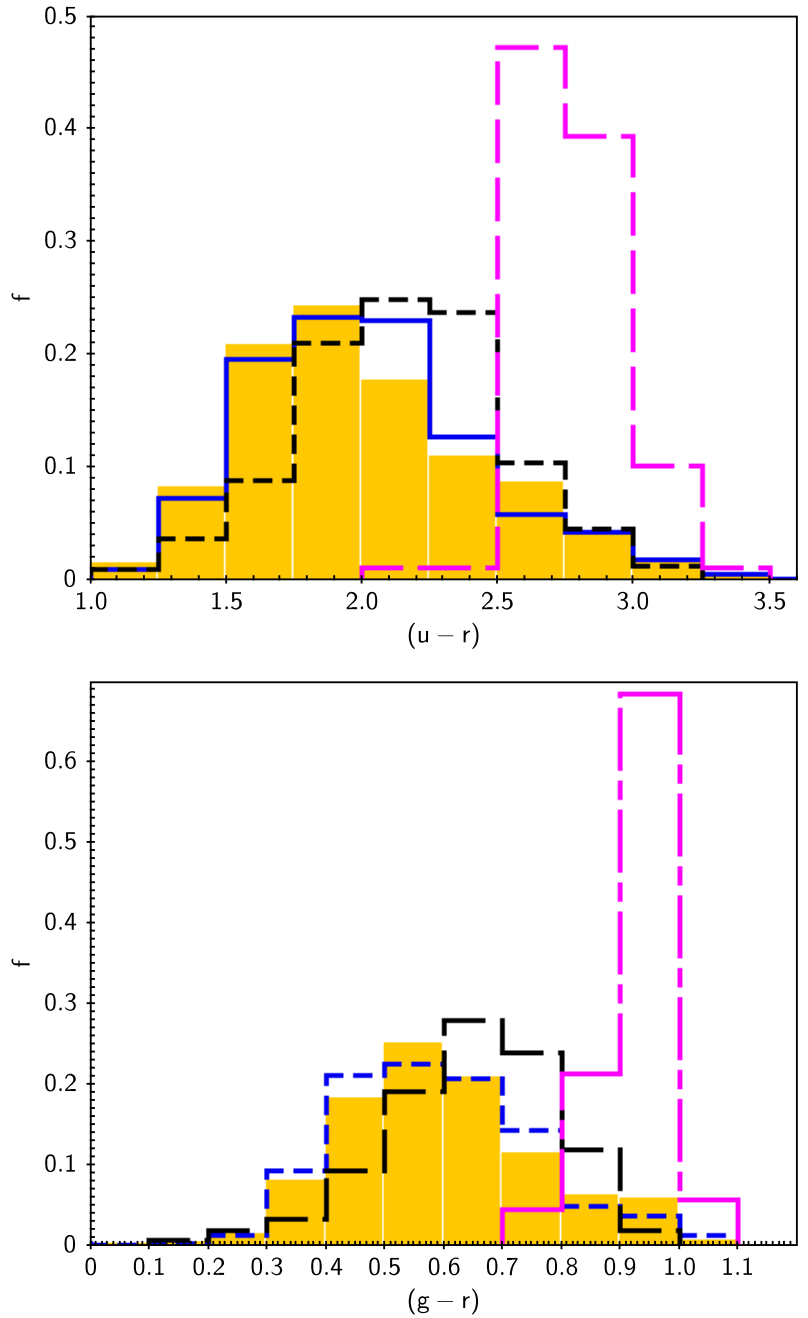

Fig. 7. Color distributions $(u-r)$ (upper panel) and $(g-r)$ (lower panel) for unbarred (shaded histograms), weak-barred (solid lines), and strong-barred galaxies in Group 1 (dot-dashed lines) and in Group 2 (dashed lines).

\subsection{Colors}

With the aim of exploring the colors of the barred galaxies with different structural strengths, in Fig. 7 we illustrate the $(u-r)$ and $(g-r)$ color distributions for different galaxy types classified previously. Strong-barred objects show a clear excess of redder colors, while unbarred and weak-barred galaxies have similar $(u-r)$ and $(g-r)$ distributions. In particular, strong bars in Group 1 show a significant fraction of host galaxies with extremely red colors $(u-r>2.0$ and $g-r>0.7)$. This finding could reflect low efficiency in star formation activity, an old stellar population, and earlier morphological galaxy types. In the same direction, Masters et al. (2011) found that red spiral galaxies have a higher fraction of bars than the blue ones in a sample obtained from the Galaxy Zoo catalog. Similarly, Oh et al. (2012) and Alonso et al. $(2013,2014)$ observed the same behavior for AGN barred hosts. Therefore, it seems that bars play an important role in the modification of the host galaxy colors, but only when this structure has become prominent enough.

In addition, in Fig. 8 we present color-magnitude diagrams for the different galaxy types. It can be seen that strong-barred galaxies are principally concentrated in the top area (red region), while unbarred and weak-barred objects are more uniformly distributed. It is clear that galaxies with strong bars in Group 
Table 3. Percentages of galaxies located above the line fitted by Masters et al. (2010ab).

\begin{tabular}{cc}
\hline \hline Galaxies & $(g-r) \geq 0.67-0.02\left(M_{r}+22\right)$ \\
\hline Unbarred & $34.82 \%$ \\
Weak-barred & $33.50 \%$ \\
Strong-barred G1 & $100.00 \%$ \\
Strong-barred G2 & $53.50 \%$ \\
\hline
\end{tabular}

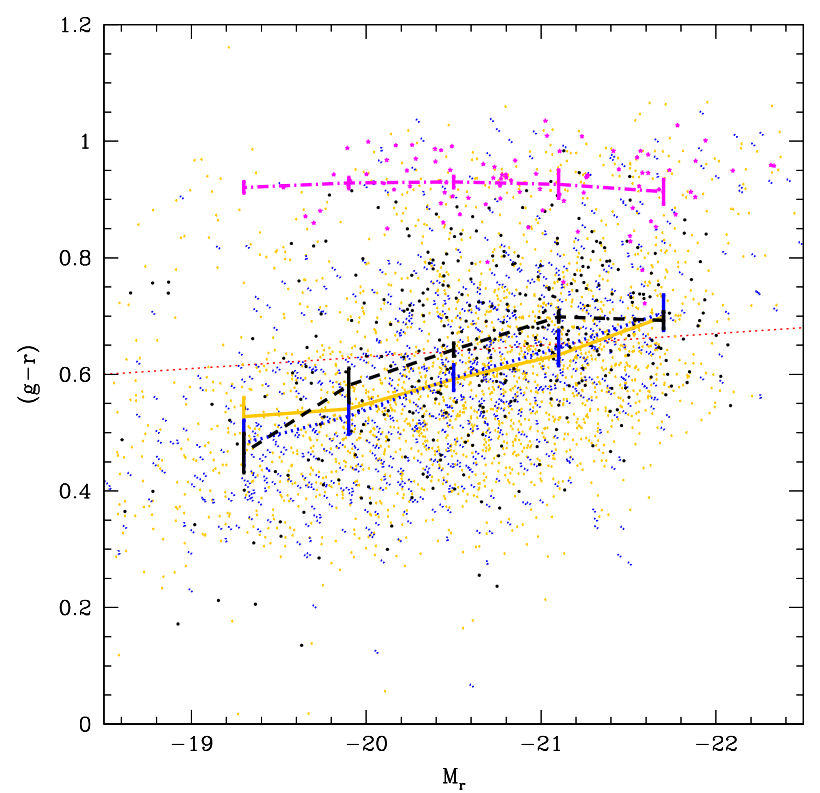

Fig. 8. Color-magnitude diagram for unbarred (orange crosses), weakbarred (blue open circles), and strong-barred galaxies in Group 1 (magenta stars) and in Group 2 (black fulled circles). The lines represent the average values for the different samples: unbarred objects (solid line), weak-barred, and strong-barred galaxies (in Group 1 and Group 2) (dotted, dot-dashed, and dashed lines, respectively). Red dashed line plots the fit obtained by Masters et al. (2010ab).

1 are located in the redder region of the color-magnitude diagram with respect to galaxies from the other samples. We have also plotted the color fit developed by Masters et al. (2010ab), which separates blue and red populations $((g-r)=0.67-$ $\left.0.02\left[M_{r}+22\right]\right)$. As we can see, galaxies with strong bars are mostly located above the line, while unbarred and weak-barred objects lies mostly below the line (blue region). This could indicate that, at the same magnitude, strong-barred galaxies are usually redder objects with respect to the other samples studied in this work. Table 3 quantifies the percentage of different galaxy types located in the red region.

Moreover, Fig. 9 illustrates the color-color diagram for unbarred, weak-barred and strong-barred galaxies in both groups. We note that the three galaxy types lie on the same straight line, although strong-barred ones are mostly grouped in the red region of the diagram (mainly those belonging to G1), while the other types show more dispersion and are more extended towards the blue region. This configuration could indicate an evolutive relation between the different classified galaxy types. In the first time unbarred disk galaxies could start forming a bar in its central region, from the instability in the disk. This bar would become increasingly prominent while it consumes gas from the disc. In the beginning, the bar would not be able to modify significantly the host galaxy characteristics. Then, when it reaches a strong prominence, it could start to affect the host galaxy, producing

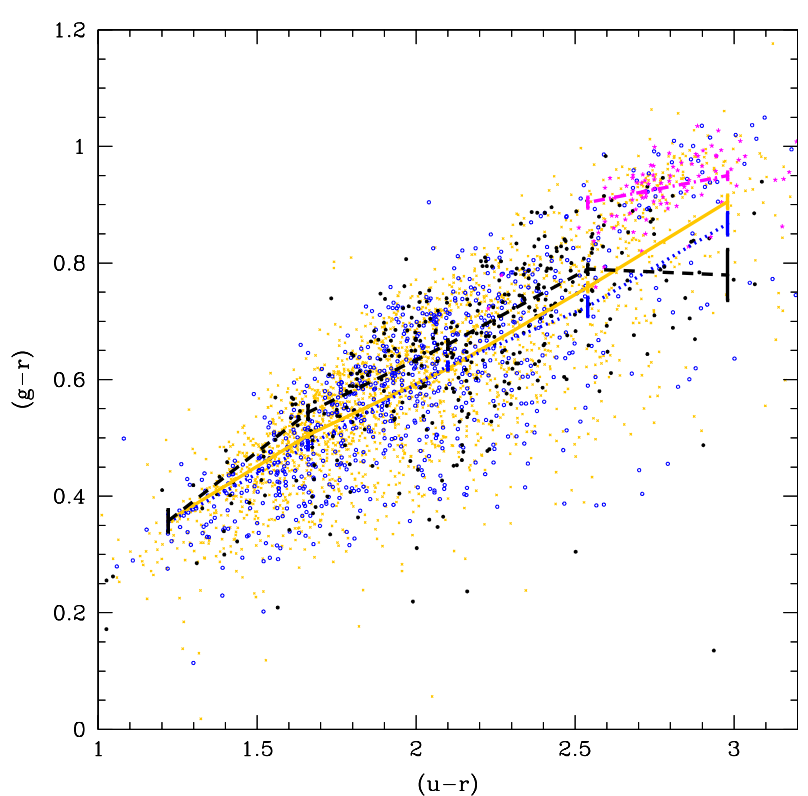

Fig. 9. Color-color diagram for unbarred disk objects (orange crosses), weak-barred (blue open circles), and strong-barred galaxies in Group 1 (magenta stars) and in Group 2 (black fulled circles). The lines represent the average values for unbarred objects (solid line), weak-barred and strong-barred galaxies (in Group 1 and Group 2) (dotted, dot-dashed, and dashed lines, respectively).

an ascent in the color-color diagram, inducing many important changes in the host galaxy properties.

Figure 10 shows the mean $(g-r)$ and $(u-r)$ colors as a function of the concentration index, $C$. It is clear that red objects increase towards the more concentrated galaxies, for the different samples. This result is consistent with expectations, since galaxies with higher values of the concentration index are related to the bulge-type morphology, and lower concentration objects to spiral galaxies. On the other hand, by evolving passively galaxies can become red without increasing concentration parameter, and also some galaxies can increase $C$ without becoming red (e.g., by merging events). It can be also seen that strong-barred galaxies are redder for the whole range of the $C$ parameter than are the counterparts of weak-barred objects and unbarred hosts in the control sample. We notice that this tendency is clearly more significant in galaxies with strong bars that belong to Group 1. In this context, the results could indicate that galaxies with strong bars could become redder than their counterparts of unbarred and weak-barred disk objects. This also supports the idea that intense bars accelerate the gas processing and is reflected in a reddened population.

\subsection{Metallicity}

The chemical features of the galaxies can store fossil records of their history of formation since they are the result of diverse physical mechanisms acting at different stages of evolution (Freeman \& Bland-Hawthorn 2002). In this sense, metallicity is one of the fundamental physical properties of galaxies; it principally reflects the amount of gas reprocessed by the stars. In addition, it depends strongly on the evolutive state of a galaxy so it is a good indicator of its age. In this analysis, as metallicity parameter, we used $12+\log (\mathrm{O} / \mathrm{H})$, which represents the ratio between oxygen and hydrogen abundances (Tremonti et al. 2004). We found that $\approx 80 \%$ of the objects in our samples 

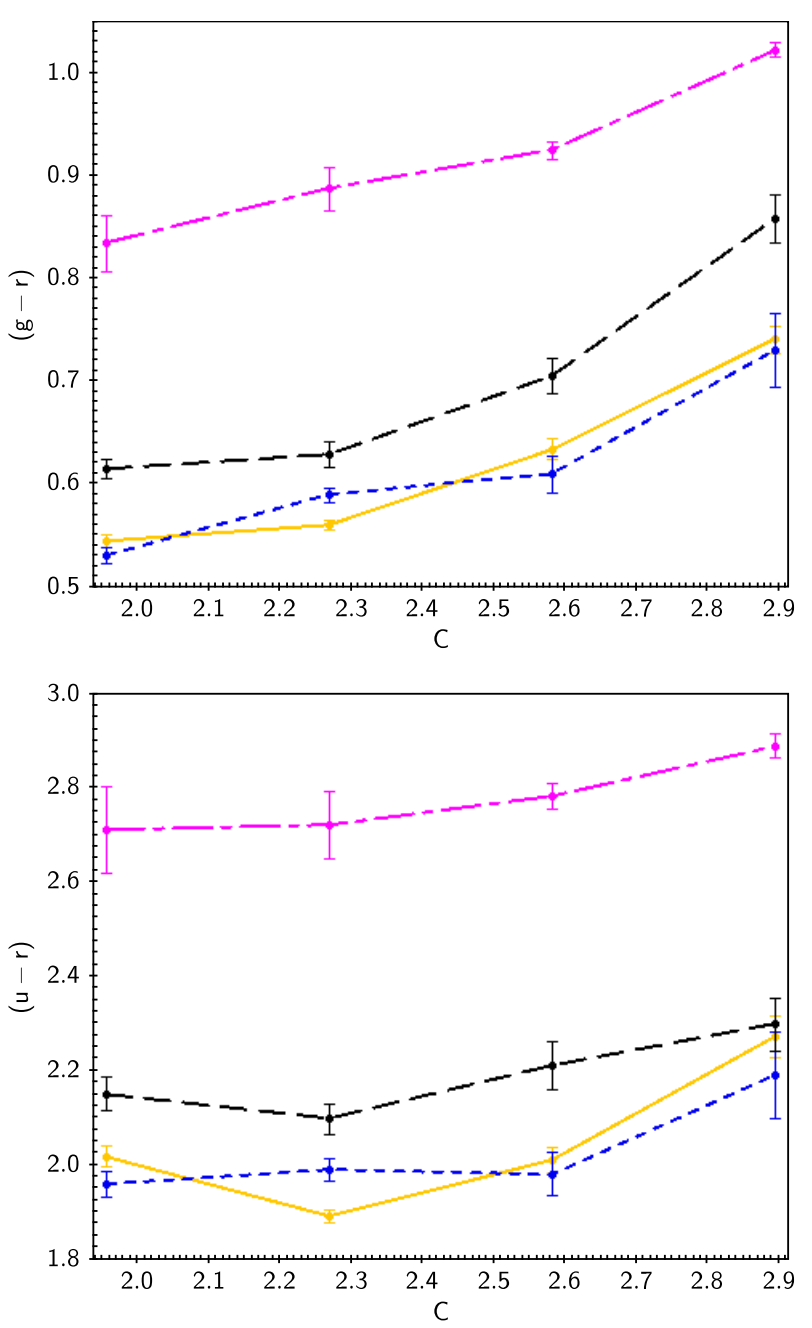

Fig. 10. Colors, $(g-r)$ (upper panel) and $(u-r)$ (lower panel), as a function of parameter $C$, for unbarred, weak-barred, and strong-barred (in Group 1 and Group 2) galaxies (solid, dotted, dot-dashed, and dashed lines, respectively).

have $12+\log (\mathrm{O} / \mathrm{H})$ measurement, and there is a null fraction of strong-barred galaxies in G1 with this parameter. Therefore, in this section, the sample of the strong-barred galaxies belongs to Group 2.

The influence of the bars in the metallicity can be seen in Fig. 11 in the histograms of the $12+\log (\mathrm{O} / \mathrm{H})$ for disk galaxies with strong and weak bars, and without bars. We also define the low and high metallicity galaxies by selecting two ranges of $12+\log (\mathrm{O} / \mathrm{H})$ values to have equal number of objects in the control sample. This threshold is $12+\log (\mathrm{O} / \mathrm{H})=9.05$. From this figure, it can be seen that strong-barred galaxies present an important excess towards high metallicity values, while unbarred and weak-barred objects show similar distributions. Table 4 quantifies the excess of disk objects with high metallicity for the different samples. This result supports the previous ones, meaning that strong-barred galaxies show low star formation activity, with older/redder stellar populations and higher gas metallicity than weak-barred and unbarred spiral galaxies. From the chemodynamical simulation studies, Martel et al. (2013) found that the chemical evolution observed within the central region of the disk galaxies depends critically on the prominence of the bar, which evolves strongly with time.

We also study the mass-metallicity relation (MZR; Lequeux et al. 1979) of barred galaxies as a tool to study the effects of
Table 4. Fraction of galaxies with metallicities higher than 9.05 .

\begin{tabular}{cc}
\hline \hline Galaxy type & $12+\log (\mathrm{O} / \mathrm{H})>9.05$ \\
\hline Strong-barred & $64.1 \%$ \\
Weak-barred & $51.4 \%$ \\
Unbarred & $50.0 \%$ \\
\hline
\end{tabular}

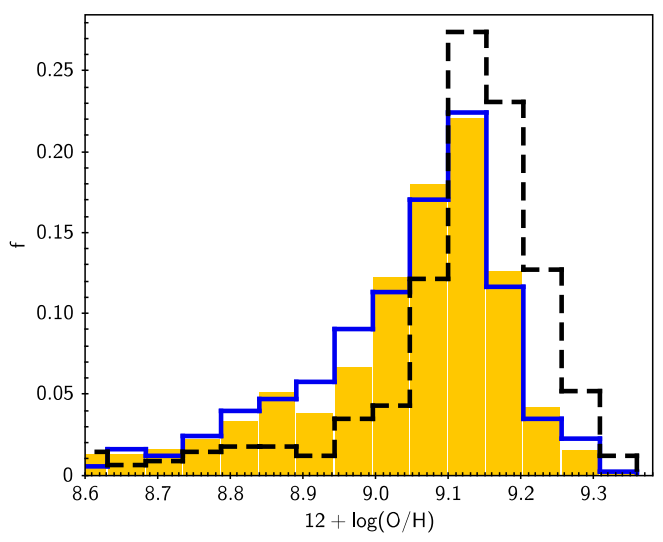

Fig. 11. Metallicity distributions for unbarred (shaded histogram), weak-barred (solid line) and strong-barred (dashed line) galaxies.

bars on the galaxy metallicity. In the local Universe, Tremonti et al. (2004) have confirmed the dependence of metallicity on stellar mass with high statistical signal. Erb et al. (2006) has extended the study to high redshift finding a similar correlation, although displaced to lower metallicity (Maiolino et al. 2007). Figure 12 shows the mass-metallicity relation for each galaxy type. We also compare these results with the results obtained by Tremonti et al. (2004), who studied the mass-metallicity relation for a sample of 53000 star-forming galaxies from SDSS. It can be seen that our galaxy samples are more metallic according to the results of Ellison et al. (2008, 2011). These authors found that the mass-metallicity relation is modulated by the star formation rate. They suggest that the metal enhancement without an accompanying increase in the star formation activity may be due to a short-lived phase of bar-triggered star formation in the past. However, the most interesting point is that strong-barred objects show a relation that falls abruptly with respect to the ones of the other samples. This tendency is more significant in low stellar mass galaxies. Nevertheless, for strong-barred galaxies with $\log \left(M_{*} / M_{\odot}\right)>10.0$ there is not a clear fall in the metallicity, and similar trends are also observed for different samples. This could indicate that prominent bars produce an accelerating effect on the gas processing and hence on the host galaxy evolution towards earlier morphological types.

In addition, it can be seen that weak-barred and unbarred galaxies do not show significant differences in the metallicity. In the same direction, this behavior is reflected in the other galaxy properties: colors, star formation activity, and stellar population. These findings could indicate that weak bars do not produce noticeable changes in the galaxy properties and the effects on the physical characteristics begin to be felt when the bar became prominent enough.

\section{Summary and conclusions}

We have performed a statistical study of physical properties of barred galaxies in contrast with unbarred ones. Our analysis is based on a sample derived from the SDSS release. We complemented these data with a by-eye classification of a sample of 


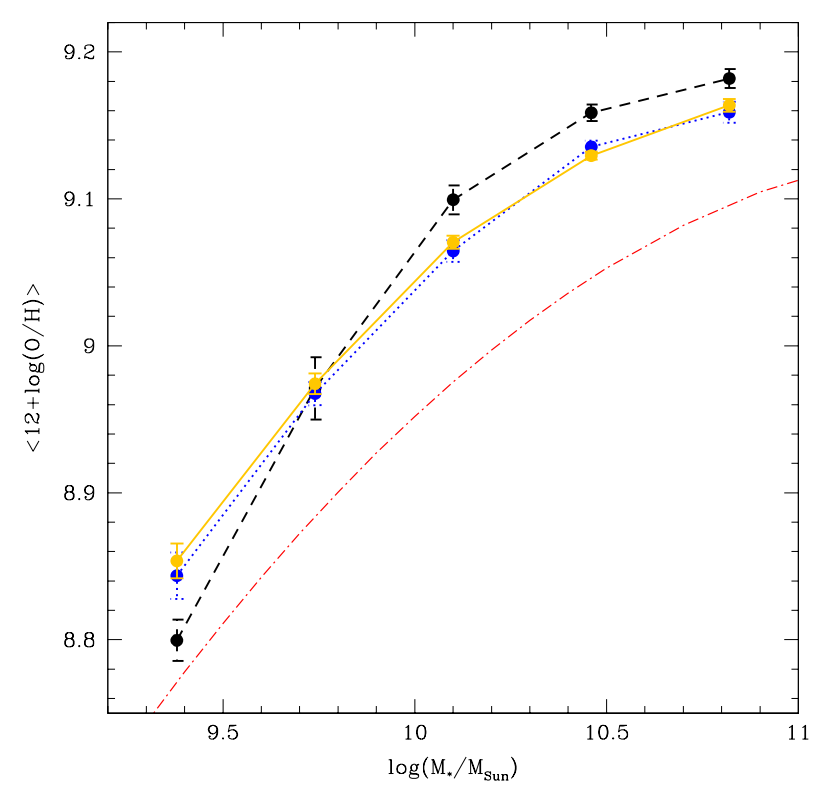

Fig. 12. Mass-metallicity relation for unbarred (solid line), weak-barred (dotted line), and strong-barred (dashed line) galaxies. The red dotted line represents the Tremonti et al. (2004) fit.

face-on spiral galaxies brighter than $g=16.5 \mathrm{mag}$, based on the presence of the bar, and taking into account the strength of the bar with respect to the structural properties of the host galaxies. In order to provide an appropriate quantification of the effects of bars on host galaxies, we also constructed a suitable control sample of unbarred galaxies with the same redshift, $r$-band magnitude, concentration index, bulge size parameter, and local environment distributions, following Alonso et al. (2013).

We can summarize the principal results of our analysis as follows:

1. We found 522 strong-barred, 770 weak-barred, and 3711 non-barred galaxies, which represents a bar fraction of $25.82 \%$ with respect to the full sample of spiral galaxies. This fraction agrees with previous studies found by other authors by visual inspection of different galaxy samples from optical images (Nilson 1973; de Vaucouleurs et al. 1991; Marinova et al. 2009; Masters et al. 2010ab; Alonso et al. 2013).

2. We observed that strong-barred galaxies show lower star formation activity and older stellar populations with respect to weak-barred and unbarred disk objects. We also found a significant fraction $(\approx 20 \%)$ of strong-barred galaxies with older stellar populations and low star formation rates that have lenticular morphology (SB0 type). This result shows that when an S0 galaxy contains a bar, it is usually a strong structure, in agreement with Aguerri et al. (2009).

3. We also studied the star formation activity and the age of stellar populations of galaxies as a function of $\log \left(M_{*}\right)$ and concentration index, $C$, in barred galaxies with weak/strong bars, and in the control sample. We found that strong-barred galaxies show a systematically less efficient star formation activity and older stellar population for different stellar mass bins, and towards earlier morphology, with respect to the other samples of galaxies with weak bars and without bars.

4. We examined the color distributions of different samples studied in this work, and we found that there is a significant excess of strong-barred host galaxies with red colors. We also found that galaxies with strong bars are redder for the whole concentration index range with respect to their counterparts of weak-barred and unbarred disk objects. In particular, for strong-barred galaxies that belong to the minor peaks of the star formation and stellar population distributions (see Fig. 3) these tendencies are clearly more significant, showing a high fraction of host galaxies with extremely red colors. These findings suggest that bar perturbations have a considerable effect in modifying galaxy colors in the host galaxies, producing an acceleration of the gas processing, when the bars became prominent enough.

5. The color-magnitude and color-color diagrams show that strong-barred galaxies are mostly grouped in the red region, while unbarred and weak-barred objects are more extended to the blue region. The positions in the color diagrams could indicate the existence of an evolutive relation between the different considered galaxy types. In this scenario, an unbarred galaxy would begin to form a bar as a consequence of a gravitational disturbance in the disk. Then, matter would fall into to the center of the galaxy, making place for a weak bar which would become gradually more prominent while the inflow accumulates material in the center. At first, the weak bar would not be able to alter significantly the host characteristics, but when this structure is strong enough, it could affect the galaxy properties significantly.

6. We also explore the metallicity, which principally reflects the amount of gas reprocessed by the stars. It shows that galaxies with strong bars present an important excess towards high metallicity values, while unbarred and weak-barred disk objects have similar distributions. The mass-metallicity relation shows that although unbarred and weak-barred galaxies are fitted by similar curves, strong-barred ones show a curve that falls abruptly. It is more important in low stellar mass galaxies $\left(\log \left(M_{*} / M_{\odot}\right)<10.0\right)$. This behavior could be suggesting that prominent bars produce an accelerating effect on the gas processing, producing significant changes in the physical properties, also reflected in the evolutionary stages of the host galaxies.

Acknowledgements. This work was partially supported by the Consejo Nacional de Investigaciones Científicas y Técnicas and the Secretaría de Ciencia y Técnica de la Universidad Nacional de San Juan. Funding for the SDSS has been provided by the Alfred P. Sloan Foundation, the Participating Institutions, the National Science Foundation, the U.S. Department of Energy, the National Aeronautics and Space Administration, the Japanese Monbukagakusho, the Max Planck Society, and the Higher Education Funding Council for England. The SDSS Web Site is http://www.sdss.org/. The SDSS is managed by the Astrophysical Research Consortium for the Participating Institutions. The participating institutions are the American Museum of Natural History, Astrophysical Institute Potsdam, University of Basel, University of Cambridge, Case Western Reserve University, University of Chicago, Drexel University, Fermilab, the Institute for Advanced Study, the Japan Participation Group, Johns Hopkins University, the Joint Institute for Nuclear Astrophysics, the Kavli Institute for Particle Astrophysics and Cosmology, the Korean Scientist Group, the Chinese Academy of Sciences (LAMOST), Los Alamos National Laboratory, the Max-PlanckInstitute for Astronomy (MPIA), the Max-Planck-Institute for Astrophysics (MPA), New Mexico State University, Ohio State University, University of Pittsburgh, University of Portsmouth, Princeton University, the United States Naval Observatory, and the University of Washington.

\section{References}

Abazajian, K. N., Adelman-McCarthy, J. K., Ageros, M. A., et al. 2009, ApJS, 182,543

Aguerri, J. A. L., \& Gonzalez-Garcia, A. C. 2009, A\&A, 494, 891

Alonso, S., Coldwell, G., \& Lambas, D. G. 2013, A\&A, 549, 141

Alonso, S., Coldwell, G., \& Lambas, D. G. 2014, A\&A, 572, A86

Athanassoula, E. 1983, IAU Symp., 100, 243

Athanassoula, E. 1992, MNRAS, 259, 345 
Athanassoula, E. 2003, MNRAS, 341, 1179

Athanassoula, E., Lambert, J., \& Dehnen, W. 2005, MNRAS, 363, 496

Balogh, M., Morris, S. L., Yee, H. K. C., Carlberg, R. G., \& Ellingson, E. 1999, ApJ, 527, 54

Balogh, M., Baldry, I. K., Nichol, R., et al. 2004, ApJ, 615, L101

Barazza, F. D., Jogee, S., \& Marinova, I. 2008, ApJ, 675, 1194

Barrow, J. D., Bhavsar, S. P., \& Sonoda, B. H. 1984, MNRAS, 210, 19

Berentzen, I., Athanassoula, E., Heller, C. H., \& Fricke, K. J. 2004, MNRAS, 347,220

Bernardi, M., Shankar, F., Hyde, J. B., et al. 2010, MNRAS, 404, 2087

Blanton, M. R., Brinkmann, J., Csabai, I., et al. 2003, AJ, 125, 2348

Bournaud, F., \& Combes, F. 2002, A\&A, 392, 83

Brinchmann, J., Charlot, S, White, S. D. M., et al. 2004, MNRAS, 351, 1151

Buta, R., \& Combes, F. 1996, Fund. Cosm. Phys., 17, 95

Chapelon, S., Contini, T., \& Davoust, E. 1999, A\&A, 345, 81

Coldwell, G., Gurovich, S., Díaz Tello, J., Soechting, I. K., \& Lambas, D. G. 2014, MNRAS, 437, 1199

Combes, F., \& Sanders, R. H. 1981, A\&A, 96, 164

Combes, F., \& Elmegreen, B. G. 1993, A\&A, 271, 391

Considere, S., Coziol, R., Contini, T., \& Davoust, E. 2000, A\&A, 356, 89

Corsini, E. M., Debattista, V. P., \& Aguerri, J. A. L. 2003, ApJ, 599, 29

Debattista, V. P., \& Sellwood, J. A. 1998, ApJ, 493, 5

Debattista, V. P., Carollo, C. M., Mayer, L., \& Moore, B. 2005, ApJ, 628, 678

Debattista, V. P., Mayer, L., Carollo, C. M., et al. 2006, ApJ, 645, 209

de Lorenzo-Caceres, A., Vazdekis, A., Aguerri, J. A. L., Corsini, E. M., \& Debattista, V. P. 2012, MNRAS, 420, 1092

de Lorenzo-Caceres, A., Falcon-Barroso, J., \& Vazdekis, A. 2013, MNRAS, 431, 2397

de Vaucouleurs, G., de Vaucouleurs A., Corwin H. G., Jr., et al. 1991, Third Reference Catalogue of Bright Galaxies (New York: Springer)

Devereux, N. A. 1987, ApJ, 323, 91

Ellison, S. L., Patton, D. R., Simard, L., \& McConnachie, A. W. 2008, ApJ, 672, 107

Ellison, S. L., Nair, P., Patton, D. R., et al. 2011, MNRAS, 416, 2182

Elmegreen, B. G., \& Elmegreen, D. M. 1985, ApJ, 288, 438

Elmegreen, B. G., \& Elmegreen, D. M. 1989, ApJ, 342, 677

Erb, D. K., Shapley, A. E., Pettini, M., et al. 2006, ApJ, 644, 813

Erwin, P. 2004, A\&A, 415, 941

Freeman, K., \& Bland-Hawthorn, J. 2002, ARA\&A, 40, 487

Friedli, D., Benz, W., \& Kennicutt, R. 1994, ApJ, 430, 105

Friedli, D., \& Benz, W. 1995, A\&A, 301, 649

Gadotti, D. A., \& de Souza, R. E. 2006, ApJS, 163, 270

Hawarden, T. G., Mountain, C. M., Leggett, S. K., \& Puxley, P. J. 1986, MNRAS, 221,41

Hawarden, T. G., Huang, J. H., \& Gu, Q. S. 1996, ASP Conf. Ser., 91, 54

Huang, J. H., Gu, Q. S., Su, H. J., et al. 1996, A\&A, 313, 13

Hummel, E., van der Hulst, J. M., Kennicutt, R. C., \& Keel, W. C. 1990, A\&A, 236,333

Ho, L. C., Filippenko, A. V., \& Sargent, W. L. W. 1997, ApJ, 487, 591

Kauffmann, G., Heckman, T. M., Tremonti, C., et al. 2003, MNRAS, 346, 1055

James, P. A., \& Percival, S. M. 2016, MNRAS, 457, 917

James, P. A., Bretherton, C. F., \& Knapen, J. H. 2009, A\&A, 501, 207

Jogee, S., Scoville, N., \& Kenney, J. D. P. 2005, ApJ, 630, 837
Kormendy, J., \& Kennicutt, R. C. 2004, ARA\&A, 42, 603

Kuehn, F., \& Ryden, B. S. 2005, ApJ, 634, 1032

Laurikainen, E., Salo, H., Buta, R., \& Knapen, J. H. 2007, MNRAS, 381, 401 Laurikainen, E., Salo, H., Buta, R., \& Knapen J. H. 2009, ApJ, 692, 34

Lee, G., Woo, J., Lee, M. G., et al. 2012, ApJ, 750, 141

Lequeux, J., Peimbert, M., Rayo, J. F., Serrano, A., \& Torres-Peimbert, S. 1979, A\&A, 80, 155

Lintott, C., Schawinski, K., Bamford, S., et al. 2011, MNRAS, 410, 166

Maiolino, R., Nagao, T., Grazian, A., et al. 2007, Il Nuovo Cimento B, 122, 935

Marinova, I., Jogee, S., Heiderman, A., et al. 2009, ApJ, 698, 1639

Martin, P. 1995, AJ, 109, 2428

Martin, P., \& Roy, J. R. 1994, ApJ, 424, 599

Martinez-Valpuesta, I., Shlosman, I., \& Heller, C. 2006, ApJ, 637, 214

Martinet, L., \& Friedli, D. 1997, A\&A, 323, 363

Masters, K. L., Nichol, R., Bamford, S., et al. 2010a, MNRAS, 404, 792

Masters, K. L., Mosleh, M., Romer, A. K., et al. 2010b, MNRAS, 405, 783

Masters, K. L., Nichol, R. C., Hoyle, B., et al. 2011, MNRAS, 411, 2026

Masters, K., Nichol, R., Haynes, M., \& Keel, W. 2012, MNRAS, 424, 2180

Martel, H., Kawata, D., \& Ellison, S. 2013, MNRAS, 431, 2560

Menéndez-Delmestre, K., Sheth, K., Schinnerer, E., Jarrett, T. H., \& Scoville, N. Z. 2007, ApJ, 657, 790

Méndez-Abreu, J., Corsini, E. M., Debattista, V. P., et al. 2008, ApJ, 679, 73

Nilson, P. 1973, Acta Universitatis Upsaliensis. Nova Acta Regiae Societatis Scientiarum Upsaliensis - Upp- sala Astronomiska Observatoriums Annaler (Uppsala: Astronomiska Observatorium )

Norman, C. A., Sellwood, J. A., \& Hasan, H. 1996, ApJ, 462, 114

Oey, M. S., \& Kennicutt, R. C. 1993, ApJ, 411, 137O

Oh, S., Oh, K., \& Yi, S. K. 2012, ApJS, 198, 40

Perez, I., Sanchez-Blazquez, P., \& Zurita, A. 2007, A\&A, 465, 9

Perez, I., Sanchez-Blazquez, P., \& Zurita, A. 2009a, A\&A, 495, 775

Perez, J., Tissera, P., \& Blaizot, J. 2009b, MNRAS, 397, 748

Pompea, S. M., \& Rieke, G. H. 1990, ApJ, 356, 416

Roberts, W. W., Jr., Huntley, J. M., \& van Albada, G. D. 1979, ApJ, 233, 67

Sakamoto, K., Okumura, S. K., Ishizuki, S., \& Scoville, N. Z. 1999, ApJ, 525, 691

Sánchez-Blázquez, P., Rosales-Ortega, F. F., \& Méndez-Abreu, J. M. 2014, A\&A, 570, A6

Sellwood, J. A., \& Wilkinson, A. 1993, Rep. Prog. Phys., 56, 173

Sellwood, J. A., \& Moore, E. M. 1999, ApJ, 510, 125

Sheth, K., Vogel, S. N., Regan, M. W., Thornley, M. D., \& Teuben, P. J. 2005, ApJ, 632, 217

Sheth, K., Elmegreen, D. M., Elmegreen, B. G., et al. 2008, ApJ, 675, 1141

Shlosman, I., Begelman, M. C., \& Frank, J. 1990, Nature, 345, 679

Skibba, R. A., Masters, K. L., Nichol, R. C., et al. 2012, MNRAS, 423, 1485

Strauss, M. A., Weinberg, D. H., Lupton, R. H., et al. 2002, AJ, 124, 1810

Tremonti, C., Heckman, T. M., Kauffmann, G., et al. 2004, ApJ, 613, 898

Vila-Costas, M. B., \& Edmunds, M. G. 1992, MNRAS, 259, 121

Weinberg, M. D. 1985, MNRAS, 213, 451

Weinzirl, T., Jogee, S., Khochfar, S., Burkert, A., \& Kormendy, J. 2009, ASP, 419,419

Zaritsky, D., Kennicutt, R. C., \& Huchra, J. P. 1994, ApJ, 420, 87

Zhou, Z., Cao, C., \& Wu, H. 2015, AJ, 149, 1 